\title{
Aerial Locomotion in Cluttered Environments
}

Dario Floreano, Jean-Christophe Zufferey, Adam Klaptocz, Jürg Germann, Mirko

Kovac

\begin{abstract}
Many environments where robots are expected to operate are cluttered with objects, walls, debris, and different horizontal and vertical structures. In this chapter, we present four design features that allow small robots to rapidly and safely move in 3 dimensions through cluttered environments: a perceptual system capable of detecting obstacles in the robot's surroundings, including the ground, with minimal computation, mass, and energy requirements; a flexible and protective framework capable of withstanding collisions and even using collisions to learn about the properties of the surroundings when light is not available; a mechanism for temporarily perching to vertical structures in order to monitor the environment or communicate with other robots before taking off again; and a self-deployment mechanism for getting in the air and perform repetitive jumps or glided flight. We conclude the chapter by suggesting future avenues for integration of multiple features within the same robotic platform.
\end{abstract}

\section{Introduction}

Many environments where robots are expected to move present complex structure, such as walls, furniture, ceilings, trees, bushes, rocks, and so forth, that we generically refer to as clutter. For example, search-for-rescue robots may be deployed in semi-collapsed buildings with debris on the ground or in forests with trees and vegetation; monitoring robots may be asked to explore buildings and houses; and environmental robots may need to disperse within urban environments to collect pollution information.

Dario Floreano, Jean-Christophe Zufferey, Adam Klaptocz, Jürg Germann

Laboratory of Intelligent Systems, EPFL Lausanne, Switzerland, e-mail: name.surname@epfl.ch

Mirko Kovac

Wyss Institute, Harvard University, Cambridge, USA e-mail: mirko.kovac@ wyss.harvard.edu 
Several articulated wheeled and legged robots have been developed for locomotion over irregular and cluttered terrain, but these robots tend to be rather slow in heavily cluttered environment and may get stuck or flip over unstable objects. The significantly longer time required to explore and find the required information may compromise the entire mission.

In this chapter, we propose to use small robots capable of moving in 3 dimensions in order to quickly and safely locomote through cluttered environments. Flight is an example of 3D locomotion that would allow robots to rapidly explore cluttered environments as long as there is an aperture sufficiently large to allow them to fly through. Here we show that although cluttered environments present several challenges for robots moving in the air, such as small size and good perception, they also present several opportunities for the robots to learn about their surroundings and pause to communicate, monitor, and save energy.

In the following sections, we present a few design features that allow small robots to rapidly and safely move in 3 dimensions through cluttered environments: a) a perceptual system capable of detecting obstacles in the robot's surroundings, including the ground, with minimal computation, mass, and energy requirements; b) a flexible and protective framework capable of withstanding collisions and even using collisions to learn about the properties of the surroundings when light is not available; c) a mechanism for temporarily perching to vertical structures in order to monitor the environment or communicate with other robots before taking off again; d) a selfdeployment mechanism for getting in the air and perform repetitive jumps or glided flight.

Each section introduces a specific feature and validates it with experimental results obtained with a custom-made robot. In the closing section, we discuss ways in which these features could be brought together within a single robotic platform in order to obtain an agile and resilient robot for locomotion in cluttered environments.

\section{Vision-based Flight}

Flight in cluttered and indoor environments brings enormous constraints in terms of size and energy because the flying platform must be lightweight to be maneuverable and small to pass through doorways or between obstacles such as buildings, posts and trees. Therefore most perceptual systems such as scanning laser range finders typically used by terrestrial robots [1] are too heavy and bulky to fit on small flying robots [2]. An alternative consists in taking inspiration from the visual system of the insect compound eye for its ability to extract visual information from an almost omnidirectional field of view with small computational and energetic requirements.

Approximately two-thirds of the neurons in the insect brain are dedicated to visual information processing [3, 4]. Biologists have unraveled a significant part of their functioning. They discovered for instance that optic flow plays a predominant role in flight control by providing information on distance to surrounding objects 
$[5,6,7]$. Interestingly optic flow can be estimated using relatively low-resolution vision sensors, which translates to small packages and limited processing needs [8].

Based on this consideration, researchers have explored what can be classified as 2D optic-flow-based control strategies. They developed autonomous systems moving on flat surfaces $[9,10,11,12,13]$, or constrained flying robots in the form of tethered helicopters [14] or horizontally flying systems $[15,16,17]$. Here instead, we aim at controlling aircraft moving in 3D and relying on roll and pitch movements in order to steer. Airplanes and helicopters in translational flight indeed use rolling and pitching movements to alter their trajectory [18].

Optic flow is the perceived visual motion of surrounding objects as projected onto the retina of an observer [19]. Assuming a mobile observer moving in an otherwise stationary environment, the motion field describing the projection of the object velocities onto its retina depends on its self-motion (amplitude and direction of rotation and translation), the distance to the surrounding objects, and the viewing directions [20]. This intricate combination of effects makes it generally difficult to extract useful information out of optic flow, especially with 3D moving systems. However, in translating aircraft, one can estimate self-rotation using rate gyroscopes, whereas translation can be assumed to be aligned with the longitudinal axis of the plane with an amplitude that can be measured by an onboard airspeed sensor (anemometer or Pitot tube). In these conditions, optic-flow can be derotated using the gyroscopic signals in order to produce an output that is proportional to the proximity of objects in the considered viewing direction $[2,18]$.

Aiming at simple 3D control strategies that can fit any small translating flying robots with limited processing power, we propose to follow a reactive paradigm where perception is directly linked to action without intermediary cognitive layers $[19,21,22,23]$. Since optic flow can be turned into proximity information as seen previously, the simplest way of achieving reactive behaviours such as obstacle avoidance, ground following or lateral stabilization is to linearly combine a set of derotated optic-flow sensors into rolling and pitching commands [24].

Such a control scheme is implemented on our 10-gram microflyer (MC2) to demonstrate fully autonomous flight in an enclosed environment. The robot is equipped with two linear camera extracting optic-flow in 3 viewing directions as shown in Fig. 1. Two rate gyroscopes provide information to derotate the optic-flow estimates in order to map them into proximity signals. A small anemometer is used to regulate the airspeed.

Figure 2 shows the coverage of the frontal field of view by the MC2 onboard cameras (left) as well as the mapping of the optic-flow values into command signals (right) for its rudder and elevator, which will directly affect the rolling and pitching rates, respectively. The gains $\Omega$ allow to tune how the plane reacts to the proximity of objects perceived in the 3 viewing directions. What is typically desired is that an object perceived in the ventral area pitches up the aircraft, which will make it climb and therefore get away from the percieved object. Similarly, the left and right weights are set so that laterally detected object will roll the aircraft away from them.

In-flight tests are carried out in a randomly textured arena of 7 by $6 \mathrm{~m}$. Once switched on, the microflyer swiftly takes off due to its airspeed controller applying 

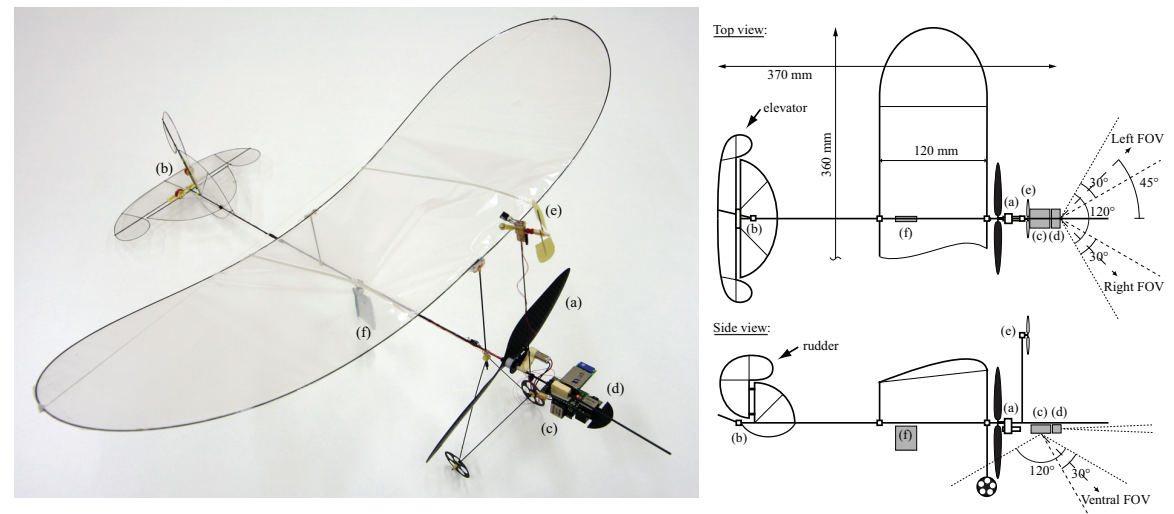

Fig. 1 The $10 \mathrm{~g} \mathrm{MC2}$ microflyer. The on-board actuators and electronics consists of (a) a $4 \mathrm{~mm}$ geared motor with a lightweight carbon-fiber propeller, (b) two magnet-in-a-coil actuators controlling the rudder and the elevator, (c) a microcontroller board with a Bluetooth wireless communication module and a ventral camera with its pitch rate gyro, (d) a front camera with its yaw rate gyro, (e) an anemometer, and (f) a 65 mAh lithium-polymer battery. Reprinted from [25].
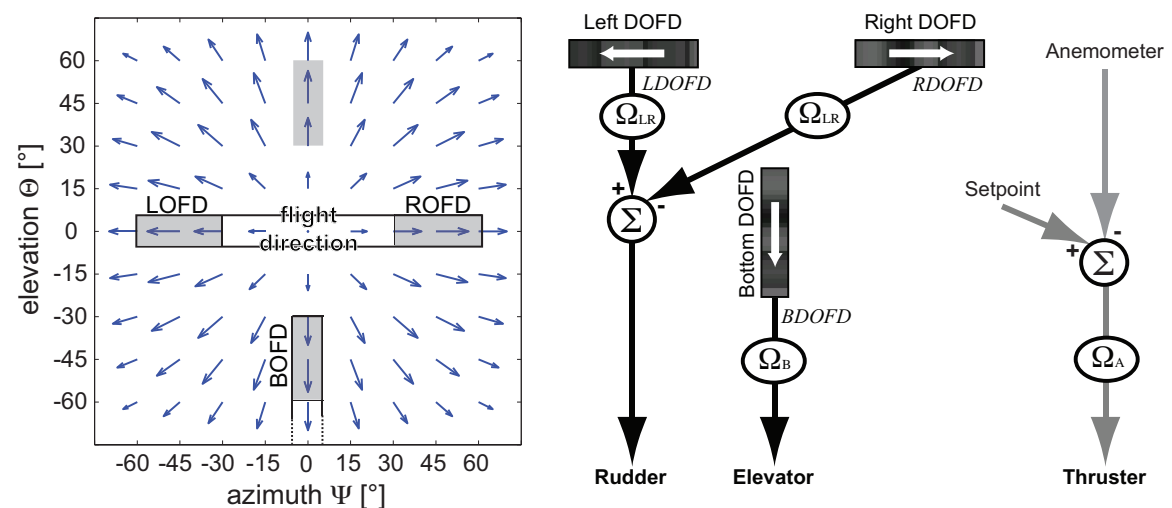

Fig. 2 Left: An azimuth-elevation graph displaying the typical optic-flow field experienced in the frontal area during straight motion. The zones covered by the cameras mounted on the MC2 are represented by the two thick rectangles. The gray zones within the thick rectangles define the three viewing directions in which optic flow is extracted. The corresponding optic-flow detectors (OFD) are prefixed with L, B, and R for left, bottom and right, respectively. A fourth OFD could have been located in the top region, but since this microflyer never flies inverted (due to its passive stability) and gravity attracts it towards the ground there is no need for sensing obstacles in the dorsal region. Right: The control strategy allowing for autonomous operation of the MC2. The three OFDs are prefixed with D to indicate that they are derotated (as explained in the text). The signals produced by the left and right DOFDs, i.e. LDOFD and RDOFD, are subtracted to control the rudder, whereas the signal from the bottom DOFD, i.e. BDOFD, directly drives the elevator. The anemometer is compared to a given setpoint to output a signal that is used to proportionally drive the engine in order to maintain airspeed reasonably constant. The $\Omega$ ellipses indicate that three gain factors are used to tune the resulting behaviour. Reprinted from [24]. 
full thrust when reading zero airspeed. Once in flight, the robot will get repelled by the ground under the effect of the ventral optic-flow detector sensing the proximity of it. As soon as a wall is perceived in one of the two lateral viewing directions, the microflyer will roll in the opposite direction. Once the aircraft is tilted, the ventral detector will not be oriented towards the ground anymore, but towards the closeby wall. It will therefore produce a pitching up reaction, which will in turn help the aircraft to steer away from the corresponding wall. As soon as the perceived proximities decrease close to zero, the microflyer will naturally get back to a level and almost straight flight as it is naturally stable by design. A video showing this autonomous behaviour is available at http://lis.epfl.ch/microflyers. More detailed description of the results can be found in [2,24].

This Braitenberg-inspired control strategy can easily be generalized to more than three viewing directions [18] in order to increase robustness by limiting the regions that are not covered by an optic-flow detector. To demonstrate how this can be done, a larger outdoor flying robot is fitted with up to 7 optic-flow detectors covering the viewing directions ranging from left to bottom to right at $45^{\circ}$ with respect to the flight direction (Fig. 3).

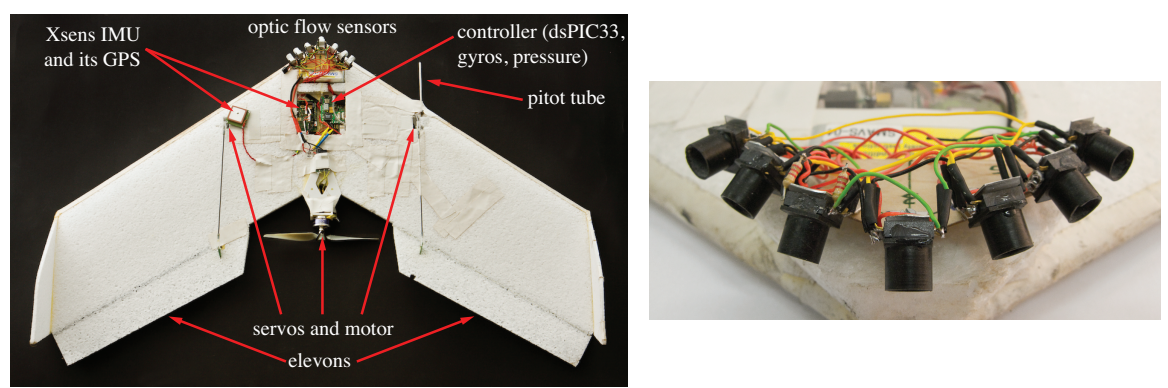

Fig. 3 Left: The swinglet flying wing used for the experiments. It has a wing span of $80 \mathrm{~cm}$ and a total weight of $400 \mathrm{~g}$ including about $50 \mathrm{~g}$ of additional sensor payload. No particular efforts have been made at this stage to reduce the weight of the sensor. Right: Visual front-end composed of seven optic computer mouse sensors pointed at $45^{\circ}$ eccentricity with respect to the aircraft roll axis. Reprinted from [18].

Here again, the basic idea of the underlying control strategy is to use weighted sums of all proximity signals coming from the various viewing directions as commands for pitching and rolling rates (Fig. 4). We name this generalized control strategy "optiPilot". The set of gains (or weight distribution) is chosen in order to achieve repulsion from all obstacles that could be sensed by any of the optic-flow detectors.

Equipped with this set of divergent optic-flow detectors, the robot is capable of taking-off automatically as it get repelled by the ground and laterally stabilized, follow the underlying terrain at a preset height depending on the strengths of the set of gains, reject lateral and longitudinal perturbations, and avoid collisions with

\footnotetext{
${ }^{1}$ Patent \# PCT/IB2008/051497 \& US 2011/0029161
} 

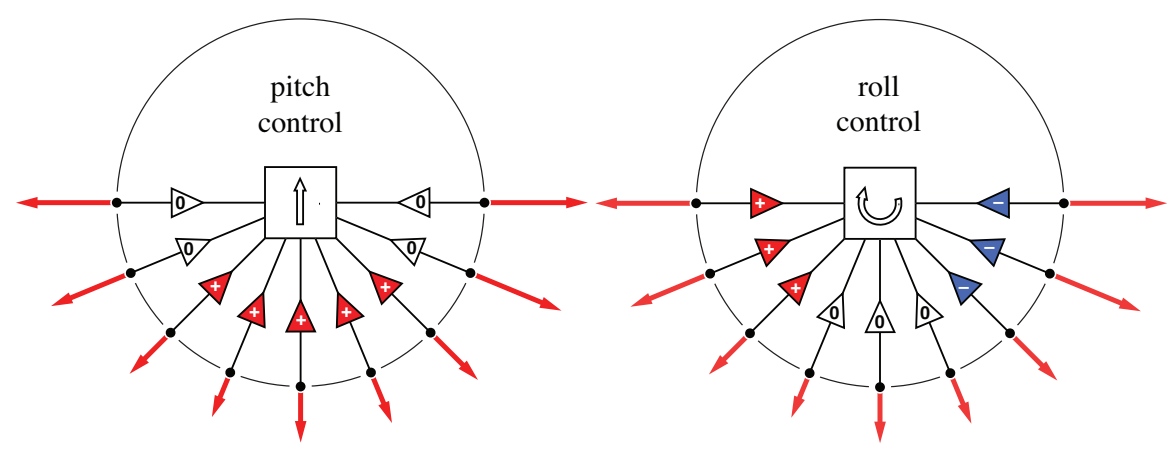

Fig. 4 Mapping translation-induced frontal optic flow (represented by the arrows in the periphery) into roll and pitch control signals. The left (resp. right) diagram represents a possible weight distribution that will make the aircraft pitch (resp. roll) away from any seen objects. The arrows in the center indicate pitch (resp. roll) direction for a positive pitch (resp. roll) signal. Reprinted from [18].

obstacles such as trees (Fig. 5), ground or water [18]. In addition, this optiPilot control strategy can be used as a low-level control layer to ensure flight stabilization and collision avoidance while a GPS-driven higher layer takes care of the trajectory control [26]. Videos of these behaviours are available for download at http://lis.epfl.ch/microflyers.
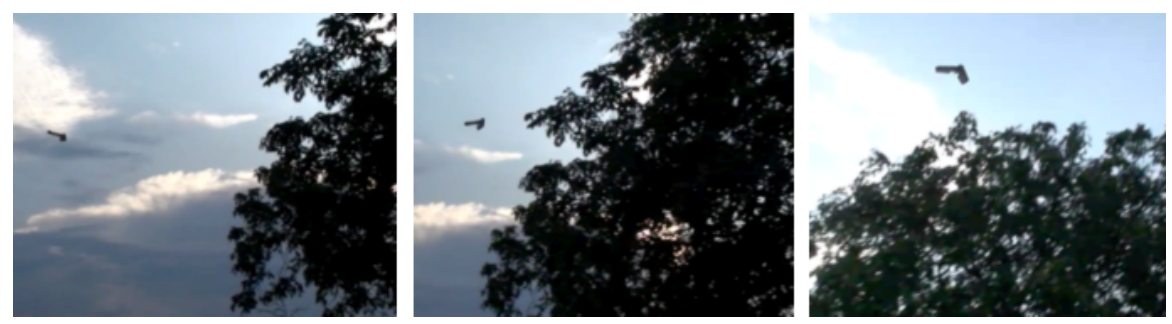

Fig. 5 Reactive tree avoidance maneuver. From left to right: swinglet approaching a tree, climbing in reaction to the perceived ventral optic flow generated by the tree, and passing above the tree.

\section{Surviving and Exploiting Collisions}

As the environment increases in clutter, space for maneuvering decreases and the risk of collisions increases. Even the best perceptual systems can fail due to lack of light or contrast for optic flow, symmetric or ambiguous information, or the small size of obstacles. Collisions are thus inevitable, but most human-made systems are not designed to withstand them. Exposed blades are especially sensitive and re- 
sult in catastrophic failures after contact. Though some recent platforms consider protecting propellers and moving parts [27, 28, 29], it is often included as an afterthought and cannot protect from large collisions. Some helicopters that can land autonomously and take off again exist [30], though only if they land on their feet. No provisions are made for landing upside-down, or for collisions with obstacles that cause loss of flight control.

Insects however have evolved resilient, lightweight and flexible bodies that allow them to frequently collide with windows or low contrast walls and continue flying. Even when falling upside-down, insects can right themselves using their legs and wings and quickly return to the air [31].

We have taken the protective and flexible bodies of insects as a design principle for a new class of flying robots that can withstand collisions, resume flight, and even fly against obstacles. Flying indoors requires small size to fit through doors and windows, and thus a maximum dimension of $40 \mathrm{~cm}$ was chosen for this design. Forward flight is useful for optic flow-based avoidance algorithms but the ability to hover is also required for maneuvering close to obstacles. To best fulfill the size and flight-mode requirements a hybrid airplane-rotorcraft design was selected that features two counter-rotating propellers for ample thrust in hover mode, an elevator and rudder for steering and a wing for forward flight and stability.

Besides the typical aerodynamic considerations that apply to all flying platforms, two additional requirements were included specific to cluttered environments:

- Collision robustness: The platform must be able to withstand collisions at full speed with hard objects such as walls. The ability to remain airborne after light contact with objects is also beneficial.

- Autonomous self-recovery: The platform can take off again after contact that results in a fall to the ground from any possible falling position without any human intervention.

These two capabilities were included through intelligent design of the robot's morphology (Fig. 6A). The teardrop-shaped wing built using a single flexible carbon fibre rod absorbs the force of frontal collisions. A second carbon fibre rod surrounds the propellers, protecting them from side impacts. Sensitive electronics, control surfaces and propellers are all housed on the main fuselage within these two protective rods, which is decoupled from the wing (and thus the force of a collision) through a spring. Using lightweight carbon fibre, mylar wings and miniature 3D-printed plastic components keeps the weight of the platform at a mere $20.5 \mathrm{~g}$, thus minimizing the kinetic energy that must be dissipated in a collision.

The shape and position of the carbon rods along with the intelligent placement of the centre of gravity (COG) are also central to the autonomous self-recovery capability. Whether the platform lands on its front (Fig. 6B-a) or on its side (Fig. 6Bb) gravity acting on the COG will always rotate the platform about its protection ring or wing to return it to takeoff position (Fig. 6B-c). The dimensioning of the different platform components and the placement of the COG is optimized to find the best balance between between aerodynamic stability and self-recovery abilities (more information on this process can be found in [32]). 

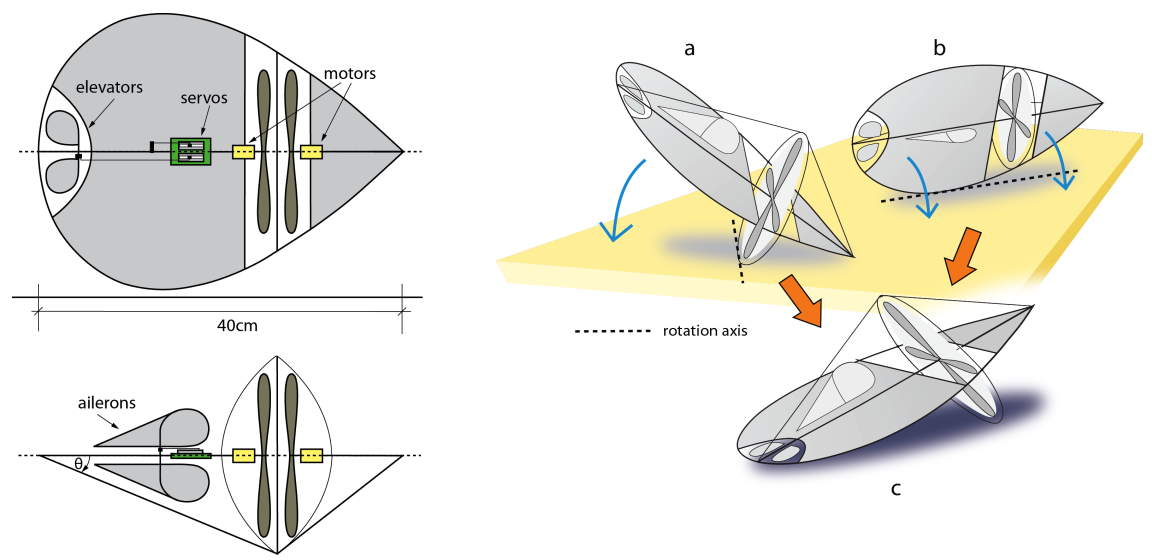

Fig. 6 Left: Initial design of platform morphology to resist collisions and to upright autonomously after a fall. Right: Passive uprighting based on platform morphology. Whether the platform falls on its front (a) or its side (b) gravity will act on its COG and subsequently place it into stable takeoff position (c). Reprinted from [32].

a

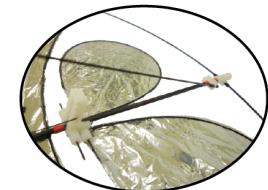

b
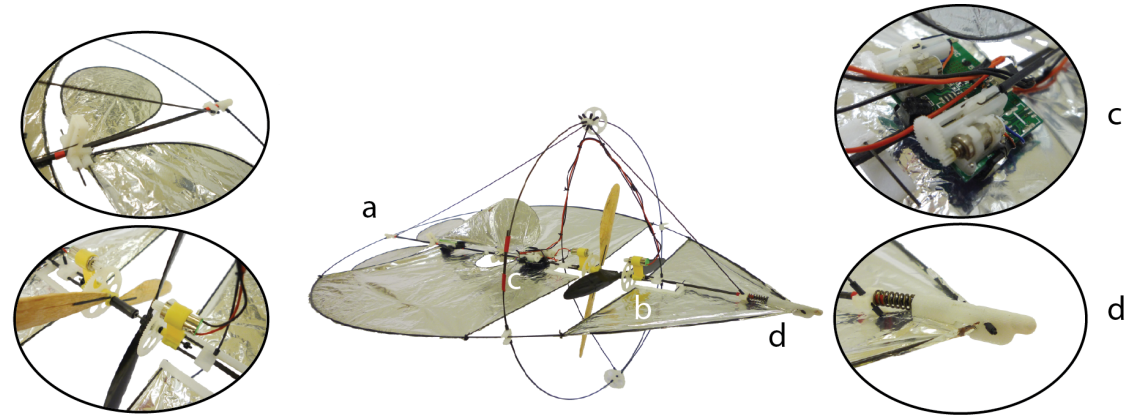

Fig. 7 The prototype flying platform with details of various subsystems. (a) depicts the linked dual elevator assembly and the connector between the wing and the back of the main bar, both printed using a 3D printer. (b) shows the coaxial motor assembly, linked using miniature ball bearings. (c) is the off-the-shelf motor-control board that features two on-board linear servos. (d) details the spring mechanism for absorbing frontal impacts and separating the wing from the main axis of the prototype. Reprinted from [32].

During remote-controlled flight tests the prototype flying platform proves to be an agile flyer in both hover and forward flight modes. The two $14 \mathrm{~mm}$ propellers are each powered by a $6 \mathrm{~mm}$ DC motor, control surfaces are actuated by two miniature servo-motors, and energy is provided by a $110 \mathrm{mAh}$ battery (enough for around 10 min of flight). Transition between the two modes is smooth and easily controllable, partially due to the low placement of the COG. During flight tests the platform was intentionally flown against walls and the ceiling during both hover and forward- 
flight modes to qualitatively assess its robustness to collisions and self-recovery capabilities. Several observations were made during these tests:

- Light contact with walls do not always cause the platform to fall to the ground. It can in fact fly along the wall, its front tip grazing the surface. This behavior resembles insects flying against a window pane looking for an exit.

- After collisions with an object that cause a fall to the ground, the prototype always settles to one of two stable positions on the ground, and in most cases can take off again without human intervention.

To further test the platform's resilience to collisions, the platform was systematically dropped from a height of $1 \mathrm{~m}$ from a variety of different starting positions. High-speed video was taken of each collision to try to analyze the deformation of the structure during a collision. As the platform hits the ground, the shock is partially absorbed by the spring at the nose of the platform (Fig. 7d), and partially by the deformation of the wing. Figure 8 shows frames from a typical collision and subsequent righting of the platform after a head-on collision with the ground.
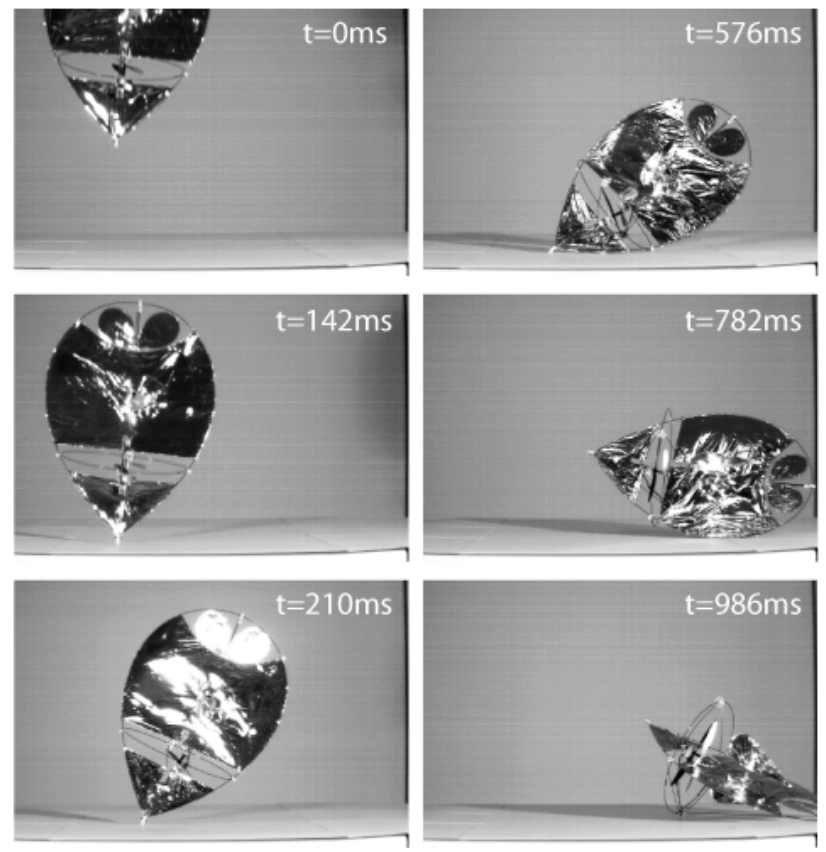

Fig. 8 Time-sequence of a typical head-on collision with the ground and subsequent self-recovery, taken with a high-speed camera. The platform rolls onto its side before rolling into takeoff position. Reprinted from [32].

The prototype presented here is a first step towards developing flying robots capable of surviving and recovering from collisions that are inevitable in cluttered environments. As with the robust exoskeletons of insects, airframe design and platform 
morphology must take into account not only aerodynamic constraints but also the ability to cope with this contact. The gravity-based self-recovery strategy presented above is only a first step and imposes severe limitations on the flight capabilities of the robot and the collisions it can recover from. As the environment gets more complicated, active recovery systems (mimicking the legs used by insects to recover when they fall on their backs [31]) will be required to push away from obstacles before taking flight.

As flying platforms become capable of surviving collisions, they can start using this contact to their advantage. Equipping the robot with a combination of sensors such as strain gages or artificial skin can allow it to detect the force of contact and gather information on its environment. This haptic information can be used to navigate in dark or low-contrast situations such as caves when other sensory modalities fail. These behaviours can be directly inspired from insects that bump into windows looking for an exit or even humans following walls with their hands in the absence of light. Attachment mechanisms can also be integrated into the platform, allowing it to perch on walls and save energy.

\section{Perching}

Power management is very important for small flying robots where the typical autonomy is in the order of 10-20 minutes and the motors consume most of the available energy [33]. Cluttered environments with vertical surfaces and ceilings offer the opportunity of temporarily perching to power off the propellers and monitor the environment or communicate with other robots from an elevated position. To date, only a few solutions exist to successfully perch for MAVs and most of them either require complex aerial maneuvers or expose the platform to high impact forces when attaching $[33,34,35,36]$. In this section, we present a perching (i.e. attachment and detachment) mechanism for MAVs that does not rely on complex control strategies but flies head-first into the substrate and dampens the impact forces to avoid potential damage to the robot when colliding (for a detailed description of the mechanism the reader may refer to [37]).

In our mechanism design we assume that the mechanism will be mounted on the tip of a flying robot, which is flying at a constant forward velocity towards a surface. The principle of the mechanism consists of two arms that are charged using a torsion spring (Fig. 9). Once the MAV impacts the surface, the spring is released by a mechanical trigger and the two arms snap forward to stick needles into the surface. In order to detach a motor pulls back two strings that are attached to the arms. Once the arms are pulled back, a small magnet fixes them in their charged position. In case that the detachment would not succeed immediately, this mechanism could discharge and recharge again several times to pull the needles out of the wall.

We dimension the torsion spring and the mass of the arms in a way that the robot is decelerated while the arms are snapping forward and has zero velocity in the moment when the needles penetrate into the surface. This is a necessary condition to 
avoid that the MAV crashes into the surface or that the snapping would bounce it off the surface.

The fabricated prototype has a total weight of $4.6 \mathrm{~g}$. We evaluate attachment to dif-
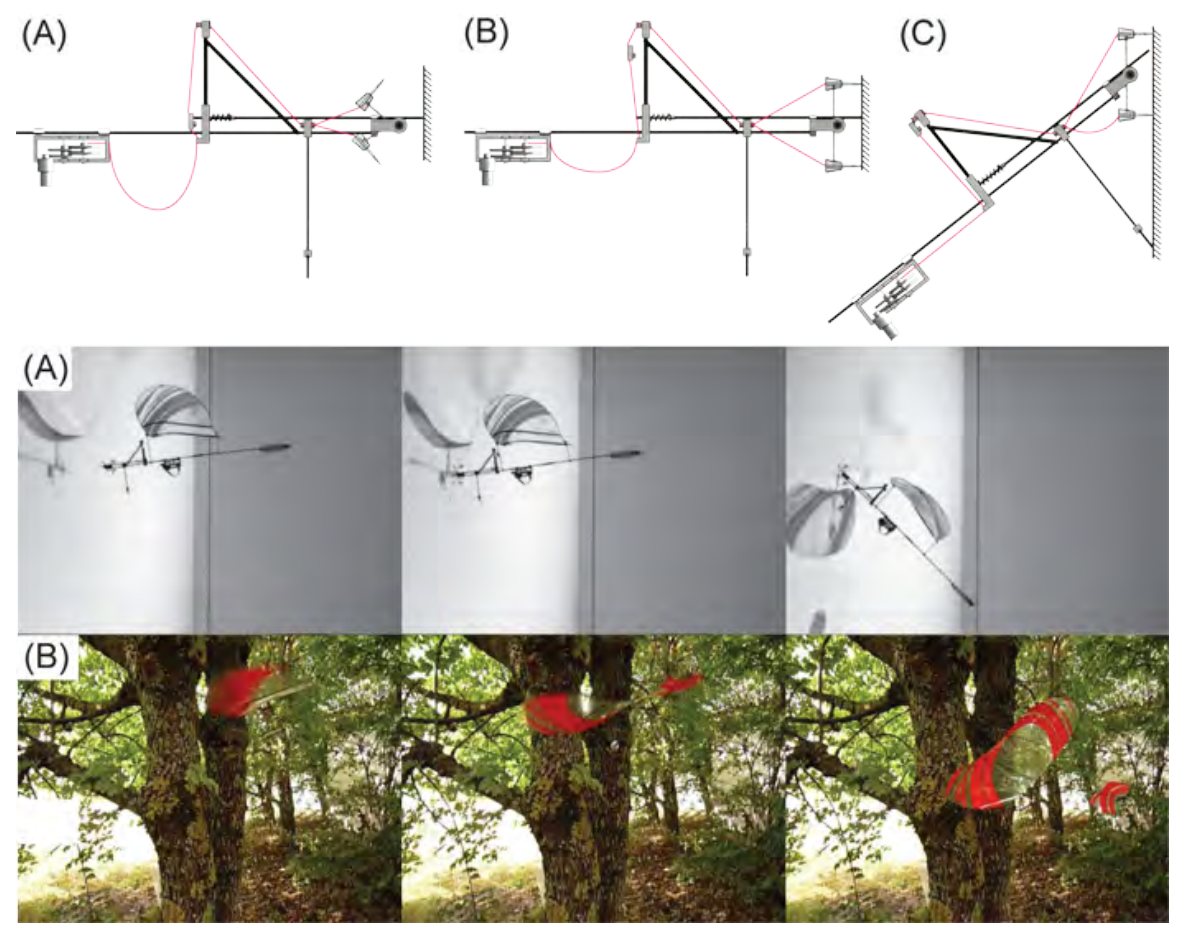

Fig. 9 Top: Attachment sequence in CAD: In flight, the perching mechanism is in a charged state; (A) once it touches the surface, the trigger separates the magnets and the arms snap forward and (B) stick the needles into the substrate; (C) finally, the mechanism settles in its stable position on the surface. Bottom: Perching sequence of the microflyer testbed to (A) a wallpaper wall, and (B) a maple tree. Reprinted from [37].

ferent substrates by launching the mechanism at painted concrete, composite hardboard wood, poplar wood and poplar bark. In order to obtain a security margin of how well the perching mechanism can support the flying robot when perched to the wall, we measure the weight that the mechanism can hold until it detaches and define the security factor (SF) to be the maximal weight divided by the weight of the mechanism. In Fig. 10 we can observe that the security factor varies from 12 to 91 and in general is lower for harder than for softer substrates.

In addition, we evaluate the reliability of the perching mechanism on the four substrates. The results in Fig. 10 show that the attachment is successful on all substrates. Also the detachment is successful in all cases, but we observe that the effort to detach is different depending on the substrate. 
To demonstrate that the perching mechanism can successfully be integrated on a MAV, we illustrate a complete perching sequence to a wallpaper wall and a maple tree in Fig. 10.

A limitation of this design is that, although the mechanism enables attachment to a
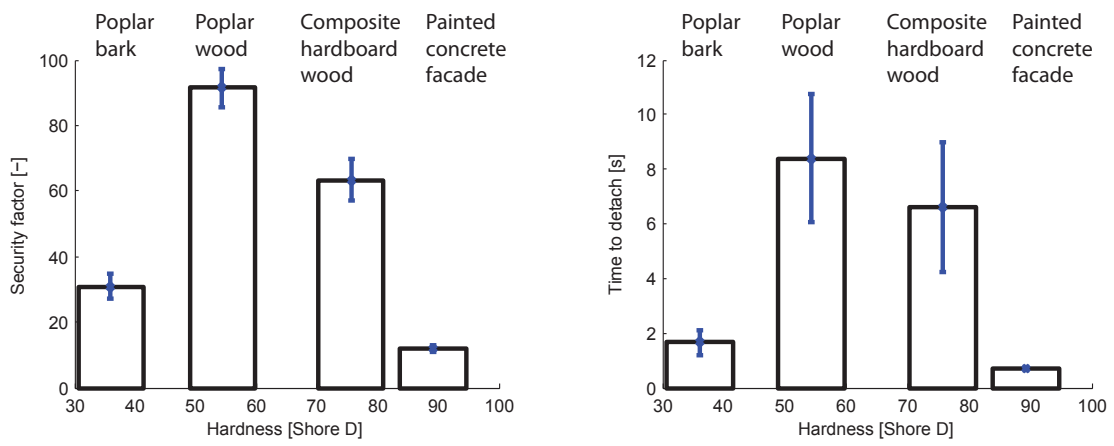

Fig. 10 Left: Security factor for attachment to four different substrates. Right: Time to detach from four different substrates. Reprinted from [37].

wide range of surfaces, it cannot perch to very hard surfaces such as glass or metal surfaces. To address this, one possible solution would be the combination of different attachment mechanisms for different situations (e.g. magnetic[33] or synthetic gecko-skin [38]).

\section{Jumping and Gliding}

As shown in the previous sections, temporarily pausing between movements can be beneficial for power management and for the ability to sense the environment for extended periods of time at a specific location. For example, the surface a small flying robot could be covered with flexible solar cells which would allow the system to recharge its batteries while on the ground and move again when it has acquired sufficient energy. Another way to move with high energetic efficiency is to adopt jumping locomotion to move on the ground. Jumping is especially adapted for small robots because the environment appears bigger when the robot decreases in size. In nature, many small animals, such as locusts, springtails, click beetles and fleas use jumping as their main means of locomotion, as it allows them to overcome relatively large obstacles despite their small body size. In robotics, a variety of jumping robots have been presented so far. For an overview on the locomotion capabilities of these robots, we summarize their jumping performance in table 1.

In this section, we describe the EPFL jumper v3 [39] which is a miniature jumping robot with a mass of $14.3 \mathrm{~g}$ that uses the same principles for repetitive jumping 
and uprighting as locusts or fleas. The main requirement in the development of the jumping mechanism is to build a lightweight propulsion unit for jumping robots, where the jumping height and take-off angle can be adjusted. For small jumping systems it is most beneficial to first slowly charge an elastic element and then use the legs as catapult to jump [40, 41, 42, 43, 44]. The working principle in our design is to charge a torsion spring using a low power motor and then release its energy to quickly extend a four bar leg linkage to perform the jumping movement.

Table 1 Performance of existing miniature jumping robots

\begin{tabular}{|c|c|c|c|c|c|c|c|}
\hline Name & mass [g] & size $[\mathrm{cm}]$ & $\begin{array}{l}\text { jump } \\
\text { height } \\
{[\mathrm{cm}]}\end{array}$ & $\left|\begin{array}{ll}\text { jump } & \text { dis- } \\
\text { tance } & {[\mathrm{cm}]}\end{array}\right|$ & $\begin{array}{ll}\text { jump } & \text { height } \\
\text { per } & \text { mass }^{a} \\
{[\mathrm{~cm} / \mathrm{g}]} & \end{array}$ & 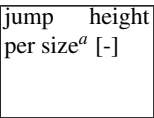 & $\mid \begin{array}{lr}\text { jump } & \text { height } \\
\text { per mass and } \\
\text { size }{ }^{a} \quad[\mathrm{~cm} / \\
\left.\left(10^{2} \cdot \mathrm{cm} \cdot \mathrm{g}\right)\right]\end{array}$ \\
\hline \multicolumn{8}{|c|}{ Class 1: Able to perform standing jumps } \\
\hline $\begin{array}{lll}\text { Closed elastica jumper } \\
{[45]}\end{array}$ & $30 *$ & 30.5 & 20 & 70 & $1.18^{*}$ & 1.16 & 3.86 \\
\hline Voice coil jumper [46] & $42 *$ & 3 & 5 & 0 & $0.12 *$ & 1.67 & 3.97 \\
\hline $\begin{array}{l}\text { Spherical crawl- } \\
\text { ing/rolling robot [47] }\end{array}$ & $5^{*}$ & 9 & 20 & 5 & $4.02 *$ & 2.23 & 44.62 \\
\hline \multicolumn{8}{|c|}{ Class 2: Able to perform standing jumps with on-board energy } \\
\hline Grillo [43] & 8 & 5 & 5 & $20^{b}$ & 1.25 & 2 & 25 \\
\hline $\begin{array}{l}\text { EPFL jumping robot v1 } \\
{[44]}\end{array}$ & 7 & 5 & 138 & 79 & 20.12 & 28.17 & 402.36 \\
\hline \multicolumn{8}{|c|}{ Class 3: Able to perform repetitive standing jumps with on-board energy } \\
\hline Microbot [48] & 11 & 46 & 38 & 0 & 3.45 & 0.83 & 7.51 \\
\hline Michigan jumper [49] & 42 & 11 & 15 & 11 & 0.37 & 1.4 & 3.36 \\
\hline $\begin{array}{l}\text { EPFL jumping robot v2 } \\
{[50]}\end{array}$ & 9.8 & 12 & 76 & 81 & 8.31 & 6.79 & 69.21 \\
\hline \multicolumn{8}{|c|}{ Class 4: Able to perform repetitive steered standing jumps with on-board energy } \\
\hline Jollbot [51] & 465 & 29.4 & 18.4 & 0 & 0.04 & 0.63 & 0.13 \\
\hline Scout [52] & 200 & 11 & 30 & 20 & 0.15 & 2.8 & 1.4 \\
\hline Mini-Whegs [53] & 190 & 10.4 & 22 & 22 & 0.12 & 2.25 & 1.18 \\
\hline $\begin{array}{l}\text { EPFL jumping robot v3 } \\
{[39]}\end{array}$ & 14.3 & 18 & 62 & 46 & 4.49 & 3.56 & 24.92 \\
\hline
\end{tabular}

The implemented jumping mechanism (Fig. 11 (a)) uses a $4 \mathrm{~mm}$ DC motor to turn a cam by way of a four stage gear box. The jumping height and take-off angle can be adjusted by adjusting the geometry of the legs. A jump can be executed every $3 \mathrm{~s}$ with a power consumption of $350 \mathrm{~mW}$. The reader may refer to [44, 39] for a more detailed explanation and characterization of the jumping principles used.

The ability to jump repetitively and to steer its jump is implemented using a carbon cage (Fig. 11 (b)) around the jumping mechanism. After landing, the jumping mechanism charges for the next jump and the cage passively uprights until the only contact with the ground is the base of the cage. Once upright, the entire jumping mechanism is inside the cage and can rotate around its vertical axis using a second DC motor around the main rod (Fig. 11 (c)).

In order to reduce the risk of damaging the legs on landing, the charging of the jumping mechanism starts already during the aerial phase to better protect the legs inside the cage. As the center of gravity is in the lower part of the structure, the robot settles in a stable upright position and is ready to steer and jump again. The motor 

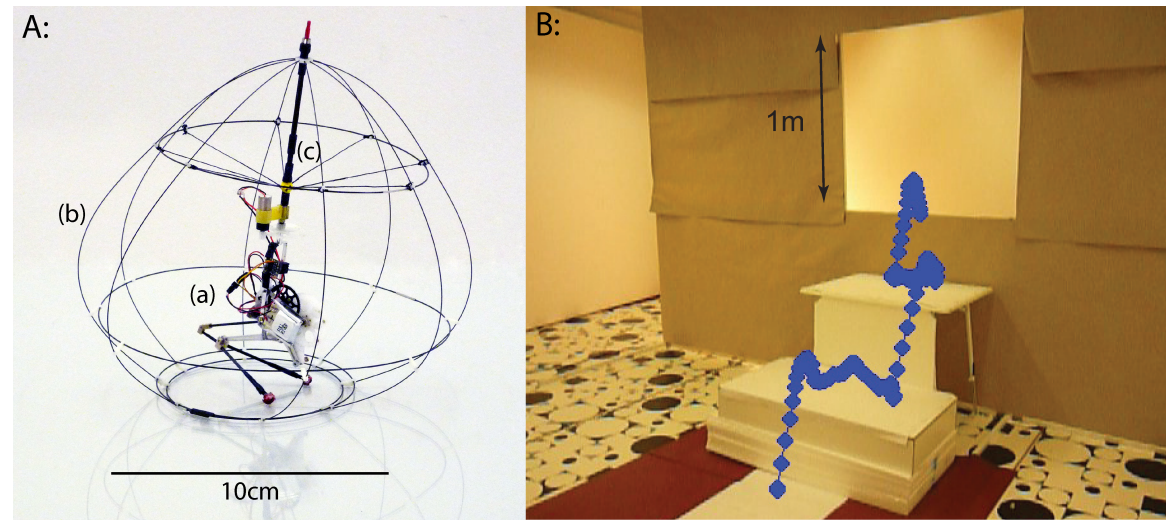

Fig. 11 A: EPFL jumper v3. (a) jumping mechanism, (b) cage, (c) main rod. B: Trajectory of the jumping robot successfully climbing two stairs of each $50 \mathrm{~cm}$ height and jumping into a window. Reprinted from [39].

to steer and the motor of the jumping mechanism are remotely controlled using a miniature infra red controller and a $10 \mathrm{mAh}$ Lithium Polymer battery. The $10 \mathrm{mAh}$ provided by this battery theoretically allow for $6.3 \mathrm{~min}$ of continuous recharging of the jumping mechanism or approximately 108 jumps. The completely functional remote controlled prototype has a maximal dimension of $18 \mathrm{~cm}$ and a mass of $14.33 \mathrm{~g}$ including batteries and electronics.

As a demonstration of the ability of our prototype to successfully perform steered jumps in cluttered environments, we build an obstacle course which consists of two stairs with a height of $45 \mathrm{~cm}$ each and a window of $1 \mathrm{~m} \times 1 \mathrm{~m}$ (Fig. 11). We place the robot on the ground at $10 \mathrm{~cm}$ distance to the first stair and aim at locomoting with sequential steered jumps up the stairs and through the window, all without human intervention on the scene. Depending on the operating skill of the human operator the window can be entered in approximately four jumps (see [39] for three successful passages of this obstacle course).

It has been suggested $[55,51,43]$ that wings could be used to prolong the flight phase of a jumping system. For lack of an existing term for this hybrid jumping and gliding locomotion, we introduce the term 'jumpgliding'. As the first miniature jumpgliding robot that is capable of successive jumpgliding locomotion without human intervention, we present the EPFL jumpglider [54] (Fig. 12 (A)), a $16.5 \mathrm{~g}$ system that can jump and transition to a steered gliding phase. Figure 12 (B) illustrates the locomotion capabilities of the EPFL jumpglider. It shows the trajectory of a jump from an elevated position of $2.53 \mathrm{~m}$ height, a stable gliding phase, three sequential jumps to progress on level terrain and finally a jump off the table to glide down to the floor.

In [56] we evaluate under which conditions the addition of wings to a jumping robot gives added benefits compared to jumping without wings. The potential benefits which are considered are (i) the ability to prolong jumps using wings and (ii) the reduction of potentially destructive impact forces that have to be absorbed by the 


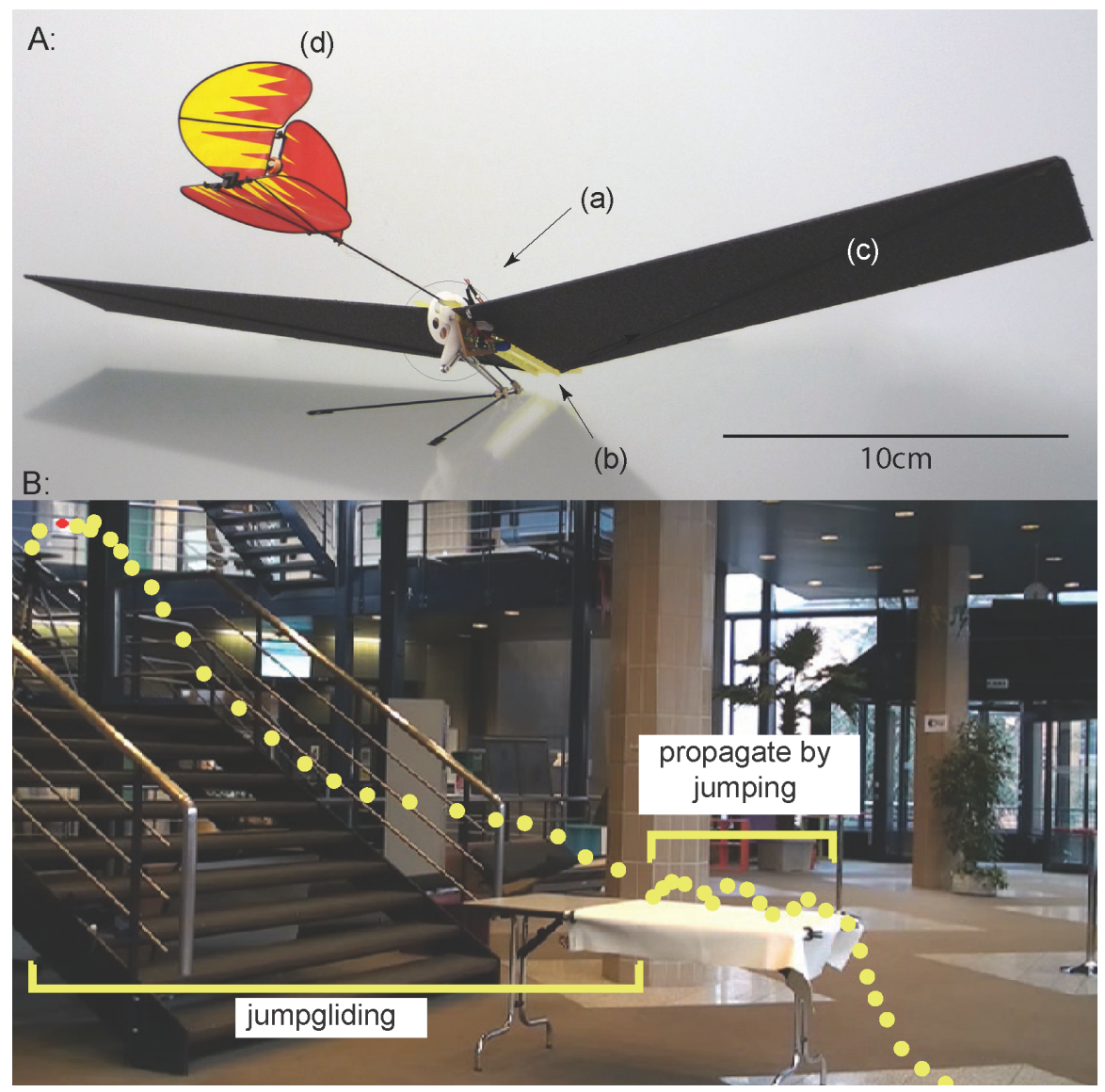

Fig. 12 A: EPFL jumpglider. (a) Jumping mechanism, (b) CNC cut Polyimide frame, (c) wings, (d) tail with rudder. B: Illustration of the locomotion capabilities of the EPFL jumpglider. Reprinted from [54].

robot structure on landing. The results indicate that wings can prolong jumps originating from elevated starting positions, but not those occurring on level ground. A jumping robot without wings, such as the EPFL jumper v3 is a better solution for locomotion on level terrain. However, wings can both reduce the impact forces and help maintain an upright orientation on landing, allowing the robot to reliably perform repetitive jumps and safely descend elevated positions and stairs. 


\section{Towards Adaptive Morphologies}

This chapter has described four features, or design principles, for small robots to move in the air in cluttered environments, such as a visual system with large field of view and integrated gyroscopes for perception and stabilization, a flexible cage for surviving and exploiting collisions, a perching mechanism to save power and increase information and communication tasks, and a self-deploying mechanism for repeated jumps or glided flight. Locomotion in cluttered environments may require adaptive forms of locomotion that can perform multiple types of locomotion, such as walking and jumping, flying and rolling, climbing and gliding, etc.

Most existing robots are designed to exploit only a single locomotion strategy, such as rolling, walking, flying, swimming, or jumping. This greatly limits their flexibility and adaptability to different environments where specific and different locomotion capabilities could be more efficient. Multi-modal locomotion capabilities could be implemented by incorporating different actuation mechanisms within the same robot. For example, the jumping and gliding robot presented here is an example of a robot with two actuation systems (one of them passive) for two locomotion modes. Another more efficient strategy would consist in achieving multiple locomotion modes with less actuation systems and motors. In order to do that, the robot could be endowed with an adaptive morphology that enables the transition between multiple states and reuse of the same actuation system for different purposes. For example, we can modify our posture and four appendices for walking, climbing, swimming, and crawling. Flexible robots, with highly integrated perceptual systems, and adaptive morphologies represent a promising solution for highly resilient and efficient robots capable of moving through cluttered, unknown, and dynamic environments.

Acknowledgements These works have been sponsored by several grants of the Swiss National Science Foundation, including the NCCR Robotics, by EPFL, and by the Science and Technology Division of Armasuisse.

\section{References}

1. R. Siegwart and D. Nourbakhsh, I.and Scaramuzza, Introduction to Autonomous Mobile Robotics, 2nd ed. MIT Press, 2011.

2. J.-C. Zufferey, Bio-inspired Flying Robots: Experimental Synthesis of Autonomous Indoor Flyers. EPFL/CRC Press, 2008.

3. N. Strausfeld, Atlas of an Insect Brain. Springer, 1976.

4. G. Taylor and H. Krapp, "Sensory systems and flight stability: What do insects measure and why," Advances in Insect Physiology, vol. 34, pp. 231-316, 2008.

5. M. Srinivasan, M. Lehrer, W. Kirchner, and S. Zhang, "Range perception through apparent image speed in freely-flying honeybees," Visual Neuroscience, vol. 6, pp. 519-535, 1991.

6. L. Tammero and M. Dickinson, "The influence of visual landscape on the free flight behavior of the fruit fly drosophila melanogaster," The Journal of Experimental Biology, vol. 205, pp. 327-343, 2002. 
7. M. Egelhaaf, R. Kern, H. Krapp, J. Kretzberg, R. Kurtz, and A. Warzechna, "Neural encoding of behaviourally relevant visual-motion information in the fly," Trends in Neurosciences, vol. 25, no. 2, pp. 96-102, 2002.

8. M. Land, "Visual acuity in insects," Annual Review of Entomology, vol. 42, pp. 147-177, 1997.

9. P. Sobey, "Active navigation with a monocular robot," Biological Cybernetics, vol. 71, pp. 433-440, 1994.

10. D. Coombs, M. Herman, T. Hong, and M. Nashman, "Real-time obstacle avoidance using central flow divergence and peripheral flow," in International Conference on Computer Vision, 1995, pp. 276-283.

11. J. Santos-Victor, G. Sandini, F. Curotto, and S. Garibaldi, "Divergent stereo for robot navigation: A step forward to a robotic bee," International Journal of Computer Vision, vol. 14, pp. 159-177, 1995.

12. M. Srinivasan, J. Chahl, K. Weber, S. Venkatesh, and H. Zhang, "Robot navigation inspired by principles of insect vision," in Field and Service Robotics, A. Zelinsky, Ed. Springer-Verlag, 1998, pp. 12-16.

13. J. Serres, F. Ruffier, S. Viollet, and N. Franceschini, "Toward optic flow regulation for wallfollowing and centring behaviours," International Journal of Advanced Robotic Systems, vol. 3, no. 27 , pp. 147-154, 2006.

14. F. Ruffier and N. Franceschini, "Optic flow regulation: the key to aircraft automatic guidance," Robotics and Autonomous Systems, vol. 50, no. 4, pp. 177-194, 2005.

15. T. Neumann and H. Blthoff, "Behavior-oriented vision for biomimetic flight control," in Proceedings of the EPSRC/BBSRC International Workshop on Biologically Inspired Robotics, 2002, pp. 196-203.

16. L. Muratet, S. Doncieux, Y. Brire, and J. Meyer, "A contribution to vision-based autonomous helicopter flight in urban environments," Robotics and Autonomous Systems, vol. 50, no. 4, pp. 195-209, 2005.

17. J. Humbert, J. K. Conroy, C. Neely, and G. Barrows, Wide-Field Integration Methods for Visuomotor Control. Springer, 2009, ch. 5, in press.

18. A. Beyeler, J. Zufferey, and D. Floreano, "Vision-based control of near-obstacle flight," $A u$ tonomous Robots, vol. 27, no. 3, pp. 201-219, 2009.

19. J. Gibson, The Perception of the Visual World. Houghton Mifflin, Boston, 1950.

20. J. Koenderink and A. van Doorn, "Facts on optic flow," Biological Cybernetics, vol. 56, pp. 247-254, 1987.

21. J. Gibson, The Ecological Approach to Visual Perception. Houghton Mifflin, Boston, 1979.

22. V. Braitenberg, Vehicles - Experiments In Synthetic Psychology. Cambridge, MA: The MIT Press, 1984.

23. A. Duchon, W. H. Warren, and L. Kaelbling, "Ecological robotics," Adaptive Behavior, vol. 6, pp. 473-507, 1998.

24. J. Zufferey, A. Beyeler, and D. Floreano, Optic Flow to Steer and Avoid Collisions in 3D. Springer, 2009, ch. 6, pp. 73-86.

25. J.-C. Zufferey, A. Klaptocz, A. Beyeler, J.-D. Nicoud, and D. Floreano, "A 10-gram visionbased flying robot," Advanced Robotics, Journal of the Robotics Society of Japan, vol. 21, no. 14, pp. 1671-1684, 2007.

26. J.-C. Zufferey, A. Beyeler, and D. Floreano, "Autonomous flight at low altitude using light sensors and little computational power," International Journal of Micro Air Vehicles, vol. 2, no. 2, pp. 107-117, 2010.

27. J. F. Roberts, T. Stirling, J.-C. Zufferey, and D. Floreano, "Quadrotor using minimal sensing for autonomous indoor flight," in European Micro Air Vehicle Conference and Flight Competition (EMAV2007), 2007.

28. P. Oh, M. Joyce, and J. Gallagher, "Designing an aerial robot for hover-and-stare surveillance," in Advanced Robotics, 2005. ICAR'05. Proceedings., 12th International Conference on. IEEE, 2005, pp. 303-308.

29. D. Schafroth, S. Bouabdallah, C. Bermes, and R. Siegwart, "From the test benches to the first prototype of the mufly micro helicopter," Journal of Intelligent and Robotic Systems, 2008. 
30. S. Saripalli, J. Montgomery, and G. Sukhatme, "Vision-based autonomous landing of an unmanned aerial vehicle," in Robotics and Automation, IEEE International Conference on, vol. 3, 2002.

31. L. Frantsevich, "Righting kinematics in beetles (insecta: Coleoptera)," Arthropod Structure and Development, vol. 33, no. 3, pp. 221-235, 2004.

32. A. Klaptocz, G. Boutinard-Rouelle, A. Briod, J.-C. Zufferey, and D. Floreano, "An indoor flying platform with collision robustness and self-recovery," in Robotics and Automation (ICRA), 2010 IEEE International Conference on. IEEE, 2010, pp. 3349-3354.

33. J. Roberts, J. Zufferey, and D. Floreano, "Energy management for indoor hovering robots," in IEEE/RSJ International Conference on Intelligent Robots and Systems (IROS2008), 2008, pp. 1242-1247.

34. M. L. Anderson, C. J. Perry, B. M. Hua, D. S. Olsen, J. R. Parcus, K. M. Pederson, and D. D. Jensen, "The sticky-pad plane and other innovative concepts for perching uavs," Proceedings of the 47th AIAA Aerospace Sciences Meeting, 2009.

35. R. Cory and R. Tedrake, "Experiments in fixed-wing uav perching," in AIAA Conference on Guidance, Navigation, and Control, 2008.

36. A. Lussier-Desbiens and M. Cutkosky, "Landing and perching on vertical surfaces with microspines for small unmanned air vehicles," Journal of Intelligent and Robotic Systems, vol. 57, no. 1, pp. 313-327, 2010.

37. M. Kovac, J. Germann, C. Hurzeler, R. Siegwart, and D. Floreano, "A perching mechanism for micro aerial vehicles," Journal of Micro-Nano Mechatronics, 2010.

38. D. Santos, B. Heyneman, S. Kim, N. Esparza, and M. R. Cutkosky, "Gecko-inspired climbing behaviors on vertical and overhanging surfaces," in IEEE International Conference on Robotics and Automation, 2008, pp. 1125-1131.

39. M. Kovac, M. Schlegel, J.-C. Zufferey, and D. Floreano, "Steerable miniature jumping robot," Autonomous Robots, vol. 28, no. 3, pp. 295-306, 2010.

40. T. J. Roberts and R. L. Marsh, "Probing the limits to muscle-powered accelerations: lessons from jumping bullfrogs," Journal of Experimental Biology, vol. 206, no. 15, pp. 2567-2580, 2003.

41. R. M. Alexander, Elastic Mechanisms in Animal Movement. Cambridge University Press, 1988.

42. M. Burrows, "Biomechanics: Froghopper insects leap to new heights," Nature, vol. 424, no. 6948, p. 509, 2003.

43. U. Scarfogliero, C. Stefanini, and P. Dario, "Design and development of the long-jumping" grillo" mini robot," in IEEE International Conference on Robotics and Automation, 2007, pp. 467-472.

44. M. Kovac, M. Fuchs, A. Guignard, J. Zufferey, and D. Floreano, "A miniature 7g jumping robot," in IEEE International Conference on Robotics and Automation (ICRA2008), 2008, pp. 373-378.

45. A. Yamada, M. Watari, H. Mochiyama, and H. Fujimoto, "A robotic catapult based on the closed elastica with a high stiffness endpoint and its application to swimming tasks," in IEEE/RSJ International Conference on Intelligent Robots and Systems, 2008, pp. 1477-1482.

46. P. Zhang and Q. Zhou, "Voice coil based hopping mechanism for microrobot," in IEEE international conference on Robotics and Automation, 2009, pp. 1783-1788.

47. Y. Sugiyama, M. Yamanaka, and S. Hirai, "Circular/spherical robots for crawling and jumping," in IEEE International Conference on Robotics and Automation, 2005, pp. 3595-3600.

48. S. Dubowsky, S. Kesner, J. S. Plante, and P. Boston, "Hopping mobility concept for search and rescue robots," Industrial Robot: An International Journal, vol. 35, no. 3, pp. 238-245, 2008.

49. J. Zhao, R. Yang, N. Xi, B. Gao, X. Fan, M. W. Mutka, and L. Xiao, "Development of a miniature self-stabilization jumping robot," in IEEE/RSJ International Conference on Intelligent Robots and Systems, 2009, pp. 2217-2222.

50. M. Kovac, M. Schlegel, J.-C. Zufferey, and D. Floreano, "A miniature jumping robot with selfrecovery capabilities," in IEEE/RSJ International Conference on Robotics and Automation, 2009, pp. 583-588. 
51. R. Armour, K. Paskins, A. Bowyer, J. F. V. Vincent, and W. Megill, "Jumping robots: a biomimetic solution to locomotion across rough terrain," Bioinspiratoin and Biomimetics Journal, vol. 2, pp. 65-82, 2007.

52. S. A. Stoeter, P. E. Rybski, and N. Papanikolopoulos, "Autonomous stair-hopping with scout robots," in IEEE/RSJ International Conference on Intelligent Robots and Systems, vol. 1, 2002, pp. 721-726.

53. J. M. Morrey, B. Lambrecht, D. Horchler, R. E. Ritzmann, and R. D. Quinn, "Highly mobile and robust small quadruped robots," in International Conference on Intelligent Robots and Systems, vol. 1, 2003, pp. 82-87.

54. M. Kovac, W. Hraiz, O. Fauria, J.-C. Zufferey, and D. Floreano, "The EPFL jumpglider: A hybrid jumping and gliding robot," in Review, 2011.

55. M. Kovac, J. Zufferey, and D. Floreano, Towards a self-deploying and gliding robot. Springer, 2009, ch. 19.

56. M. Kovac, J.-C. Zufferey, and D. Floreano, "Hybrid jumping and gliding locomotion for miniature robotics," In review, 2011. 